\title{
Multi-object Tracking In The Overlapping Area Based on Optical Flows
}

\author{
Ningning Wang ${ }^{1, a}$, Mingli Luo ${ }^{2, b}$ and Xiaohui Luo ${ }^{3, c}$ \\ ${ }^{123}$ College of Mathematics and Computer Xihua University, Chengdu, 610039, China \\ a18228019493@163.com, 'b13558819887@163.com, 'Ixh10801@163.com
}

Keywords: Lucas-Kanade optical flow, partial occlusion and interaction area, multi-target tracking

\begin{abstract}
For the question of incorrect target tracking when a dynamic occlusion occurred between two or more objects within a surveillance video, this paper raises use the optical flow to track pedestrians in partial occlusion and interaction area.We based single target locating and tracking model on the optical flow field of the same target has globally consistent.There are two cases of multiple target tracking in the occluded areas:(1)if the direction of moving objects were too different,the direction can be used as constraints and updated object template to ensure the track process continuity;(2)When they were similiar,the position of target is predicted by its optical flow speed and direction,by using the combination of this two cases, the algorithm can provide real-time and robust multi-object tracking in the occluded areas.
\end{abstract}

\section{Introduction}

Moving targets tracking under occlusionis is a difficult point in targets detecting and tracking.The key to slove this question is to establish its proper tracking model,usually the established model based on appearance and edge patterns may become inaccurate during or after the occlusion depending on changes in the lighting or the clothing color.The motion estimation between video frames by optic flow can get the motion parameters of the moving object(speed direction、acceleration), according to the optical flow field of the same target has globally consistent,and different targets have different speed and optical flow direction,we establish the objective model and updated object template in obstructed place.

There are many occluded tracking algorithms based on appearance and edge patterns ,[1]makes likelihood calculation by using new DG_CENTRIST feature and color feature,and then calculates the overlapping ratio of the tracking object and the object in the current frame using coincidence degree to measure the occlusion. Rowe et al. [2] propose weighted fragment based mean shift with Kalman filter with the consideration of target color feature.[3] propose an appearance model built by attributed relational graph (ARG),to analyze tracking situations in current frame using a combination of pixel colour and spatial information. Particle filters provide another means of tracking objects through occlusions,[5] can capable of tracking multiple targets reliably combine the scale invariant feature transform (SIFT)method with color based particle filter algorithm.

For motion based methods,the tracking is conducted according to the motion field.Haritaoglu et al. [6] proposed using the results of motion segmentation directly,by searching for maxima in the vertical projection of the motion region containing the people of interest,but failure when people occlude one another vertically.[8]Real-time multiple objects tracking with occlusion handling in dynamic scenes using a combination of object segmentation, and merging and splitting detection.[9]utilizing color,optical flow and depth clues incorporate the track framework, and the optical flow can get a rough estimation of the target location . Simon et al.[10]have proposed using optical flow discontinuities to segment groups and resolve occlusion,the ratio of continuous to discontinuous pixels within a region can be used to locate the overlapping edges, and incorporate this into object tracking.[11] proposes an occlusion detection algorithm based on the KLT (Kanade-Lucas-Tomasi) method and color information,combination with particle filter tracking for Human Tracking.[4]propose sparse occlusion detection with optical flow, the task can be posed as a variational optimization problem, and its solution approximated using convex minimization.[12]use the landmark points to represent the moving objects and the movement of these points is estimated 
using a sparse optical-flow method. combination with adaptive filters and neural networks for object tracking.Paper content is as follows:Section 2 describes how to establish the single target tracking model;Section 3 sloves mutli-target tracking problems in the occulded area;Section 4 shows the experimental results and data;Finally, Section 5 gives the conclusions and possible further directions.

\section{Single objective model and tracking based on optical flows}

Estimating motion between video frames by lucas-kanade optic flow method,and obtaining the motion region by Gaussian mixture model,use the foreground as a constraint for getting the optical flow direction and speed.Getting the main direction by normalized simplification model,and using the learning energy results in accordance with main direction to establish the object model.The algorithm framework is shown as Fig.1.

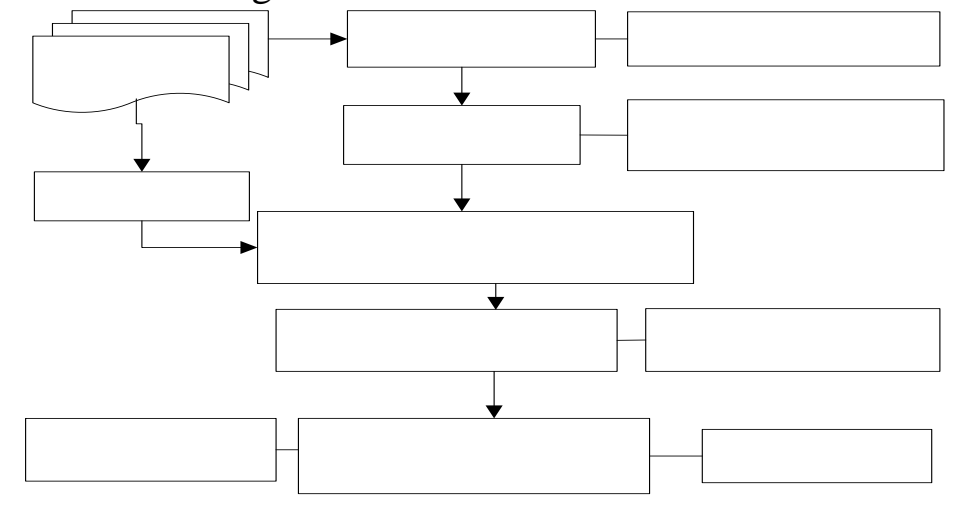

Fig. 1. The framework of single target tracking algorithm

\section{Lucas-Kanade optical flow estimation}

The key of target tracking method based on optical flow method is to get accurate motion estimation.The optical flow formulation was first proposed by Horn and Schunck (1981)[7].[13,14]is the evaluation of optical flow computation method.More commonly we view opaque objects offinite size undergoing rigid motion or deformation. In this case neighboring points on the objects have similar velocities,so we adopt Lucas-Kanade optical flows[15],to avoid the aperture problem.Experiments showed that this algorithm has lower complexity and better performance.

The optical flow estimation of pixel within motion object region

Background modeling for consecutive frames was based on mixture Gaussian model,then the object is obtained by the morphological operation for foreground segmentation result,locating it with finding contours.As a constraint only the pixels in the box are for processing. We follow the code in [16] to visualize a flow field as Fig.2 (a)(c) .

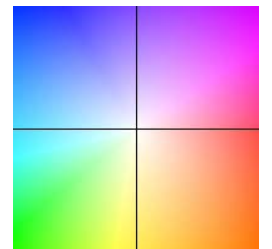

(a) The visualization of flow fields

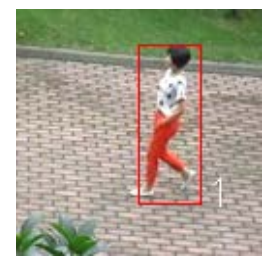

(b) Single object

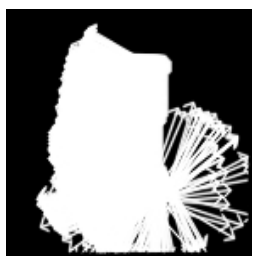

(c) flow fields

Fig. 2. The visualization of flow fields: each pixel denotes a flow vector where the orientation and magnitude are represented by the hue and saturation of the pixel, respectively.

Optical flow direction normalized simplification processing within moving target

The changing pattern of hands and legs when pedestrian moving likes fan shape or otherwise,in order to get the main motion orientation,the normalized simplification processing procedure is applied to optical flow direction within moving target.The normalized model can be divided into the following types:(1) big scene,small target,the optical flow direction is divided into eight areas like Fig.3 (a),the range of each is 45 degrees,and Numbers represent the direction.(2) small scene, big target, we divide it into sixteen areas as shown in Fig.3 (b),the range of each is 22.5 degrees.(3)is also a small scene,considering the target motion direction may be between two regions in Fig.3 (b),so we will have two neighboring areas in Fig.3 (b) merged into a new model and visualize it in Fig.3(c). 
(a)

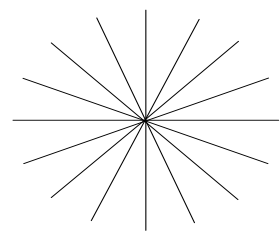

(b)

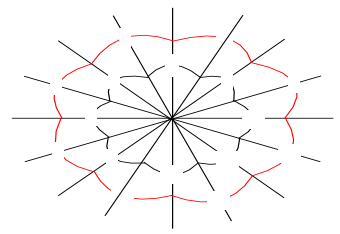

(c)

Fig. 3. Optical flow direction normalized model within moving target in different scene

Based on the above normalized model,making the energy statistics of targets in each direction $D_{k}$, and the result is :

$M_{D}\left(D_{k}\right)=n_{k}, k=0,1,2 \ldots . L-1$

$n_{k}$ is the number of pixels belong to $D_{k}$, L is the possible direction of object,then we can get the energy histogram of object.As shown in Fig.4 (a) is the energy histogram of moving object in Fig.2 (b) and its normalized model can be visualized in Fig.3 (c).We specified the direction of the largest energy is the target motion direction $\mathrm{D}$, and the energy of current object is $M_{D}$, The optical flow field of object in Fig.2 (b) after normalized simplification procedure as shown in Fig.4 (b).Based on a lot of experimental observation we can conform that the velocities of object in accordance with optical flow principal direction are basically identical.
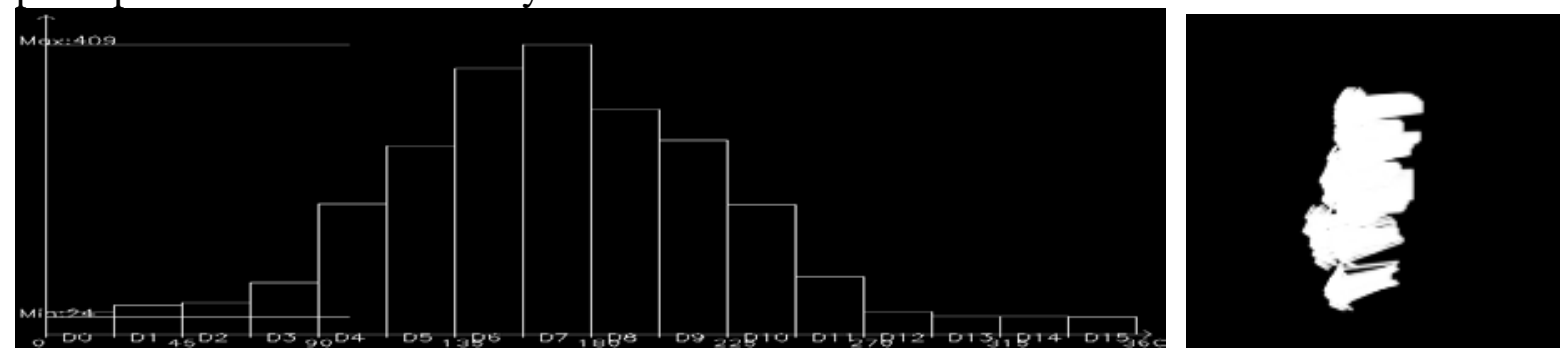

Fig. $4 . \quad$ (a) object energy histogram

(b) normalized simplification result

Target model establishment and tracking

Learning the energy of each drection of moving object in current frame $M_{D}\left(D_{k}\right)_{i}$, and updating its energy range:

$$
\begin{aligned}
& M_{\text {min }}=\min \left(M_{D}\left(D_{k}\right)_{1}, M_{D}\left(D_{k}\right)_{2} \ldots M_{D}\left(D_{k}\right)_{i}\right), i=1,2 \ldots V . \\
& M_{\max }=\max \left(M_{D}\left(D_{k}\right)_{1}, M_{D}\left(D_{k}\right)_{2} \ldots M_{D}\left(D_{k}\right)_{i}\right), i=1,2 \ldots V .
\end{aligned}
$$

$\mathrm{V}$ represents the number of learning vector is 10. Learning the width and height of 10 detection results and getting its average FrameW and FrameH. Learning the target moving directions:

$$
D=\sum_{i=1}^{10} m_{i} D_{i} / \sum_{i=1}^{10} m_{i} \cdot
$$

The $m_{i}$ is weight, the closer the current frame, the bigger the weight.Get the target motion direction of the current frame $D_{i}$, if $D_{i}=D \pm 2$, update D. When an object matches, using target moving direction as constraints,the detection and target box is used to determing the size of search box,shifting in the search box and determining the target location by the energy similarity values ,the energy range of current object must between $M_{\min }$ and $M_{\max }$. The single target tracking results of using the above algorithm is as shown in Fig.2 (b).

\section{Multi-object tracking in partial occlusion and interaction area}

On the basis of single target tracking method,there are two cases of multiple target tracking in the occluded areas:(1) the direction of moving objects were too different,the features of the former are a simple,and stable, but the latter are a complex, and limited;(2) the direction of moving objects were similiar;

The pedestrian block tracking based on real-time target model updating

When targets moving direction have big difference as Fig.5 (a)(c),the features of the former are stable,the energy of the latter becomes smaller with more occuled areas,as the change of red area in Fig.5 (b) (d).At this time we no longer learn the target box size,on the basis of single target tracking 
method,combine with real-time updates of energy feature $M_{D}$, and achieve the pedestrian tracking in partial occlusion and interaction area.

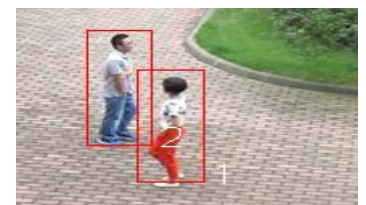

(a)objects unobstruction (b)optical flow field

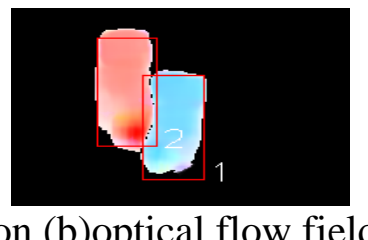

Fig .5.

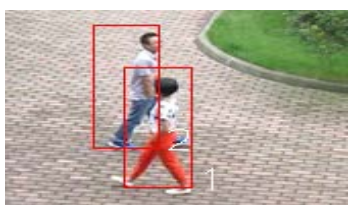

(c) targets occlusion

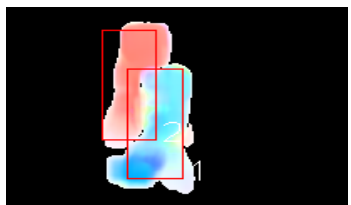

(d)optical flow field

Object tracking based on the prediction of its postion by optical flow speed and direction

When little difference between target motion direction as Fig.6 (a)(c), what we get the optical flow direction statistics of pixels is within objects and its overlap area,as shown in Figure 6 (b) (d),the energy of rectangular box 1,2 are simliar,with 3.1 method can lead to disorderly matching problem.In this paper,we adopt its optical flow speed and direction to predict the position of occlued target .
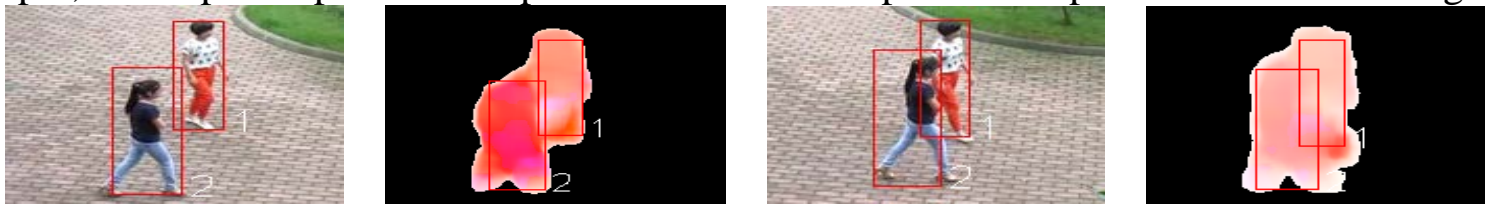

Fig .6. (a) objects unobstruction (b)optical flow field (c) targets occlusion(d)optical flow field

With the acquired optical flow values for every pixel in the object, the new location of the target in the next frame can be estimated .Keep on learning and updating the horizontal velocity u and vertical velocity $\mathrm{v}$ of the object:

$$
\begin{aligned}
& u=\sum_{i \in M} F_{x}(i) / \text { PixNumInN . } \\
& v=\sum_{i \in M} F_{y}(i) / \text { PixNumInN . }
\end{aligned}
$$

where $F_{x}(i)$ and $F_{y}(i)$ are the optical flow of pixel $i$ in the horizontal and vertical directions respectively,PixNumInN is the number of pixels that meet the main direction within object,and stop learning when objects occlusion.Suppose the center of object $M$ in current frame $t$ is located at $\left(x_{t}, y_{t}\right)$.Then its position in nextframe $\mathrm{t}+1$ is calculated as $\left(x_{t+1}, y_{t+1}\right)=\left(x_{t}+d x, y_{t}+d y\right)$, where $\mathrm{dx}$ and dy are the displacements estimated by averaging the learning velocity $\mathrm{u}, \mathrm{v}$ of 10 frames.This procedure is demonstrated in Fig. 7.The following search then starts from $\left(x_{t+1}, y_{t+1}\right)$. The center of $\mathrm{M}$ is allowed to shift within a neighborhood region (the gray search region in Fig.7).To further incorporate the scale changes during tracking,the bounding box of candidate target is allowed with an adaptive enlarging or shrinking.But to ensure a reasonable appearance change between adjacent frames,the scale change is suppressed by a maximum of $10 \%$ in the horizontal and vertical directions.
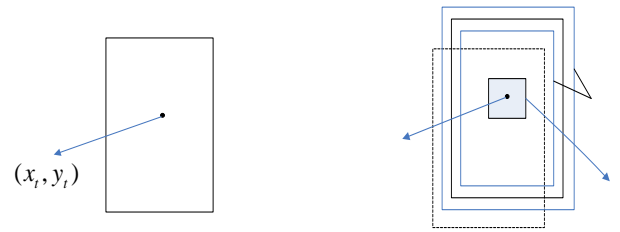

Fig. 7. The prediction of object postion by optical flow speed and direction

Multi-object tracking based on the above method

For Multi-object tracking in occuled area, we can use the direction as a constraint and updated the object template timely and make a combination with predict its location by optical flow speed and direction.As shown in Fig. 8(c) (d),target 1, 2 have the similar color but differ with 3.By means of the algorithm proposed by paper, the results of occlusion tracking are as shown in figure 8 (a) (b). 


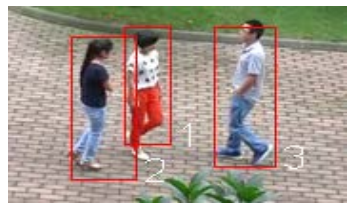

(a) unobstructed objects (b) targets occlusion

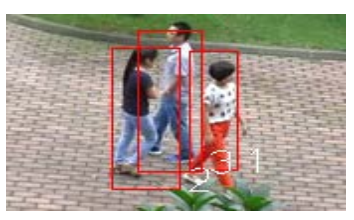

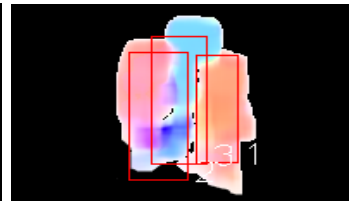

(d)optical flow field

Fig.8. Multi-object tracking based on the above method

\section{Experiments and analysis of the experimental data}

We respectively adopted different target moving direction of video clips under different scenarios for tracking,each image (a) is the panorama before temporal occlusion,(b)is the tracking result in occlude areas, (c)is the result when they separated from themslives.Figure 9 were two targets occlusion tracking results under the scenario 3. Figure 10,11 were multi-target occlusion tracking results under the scenario 1 and 2.Scenario 2, 3 compared with scenario 1, with dark illumination and dull colouring.

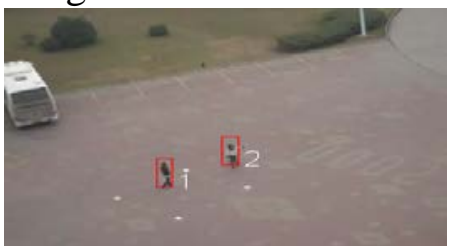

Fig.9. (a) frame 138

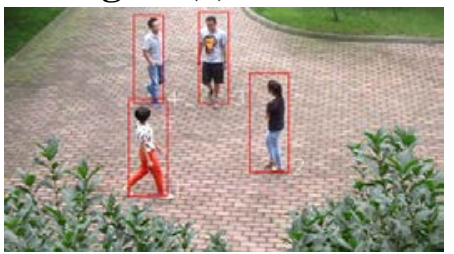

Fig.10. (a) frame 174

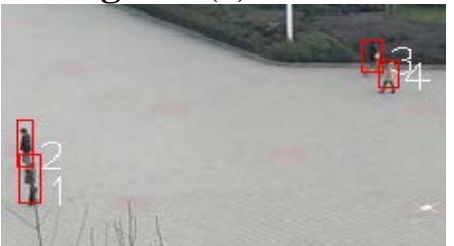

Fig.11. (a) frame 130

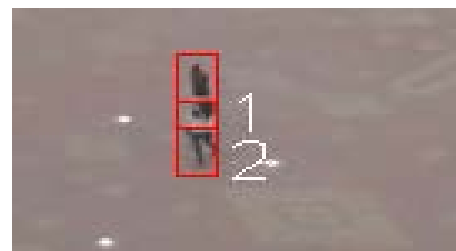

(b) frame 181

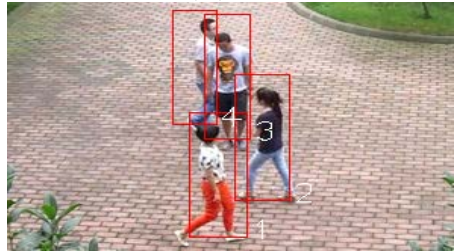

(b) frame 180

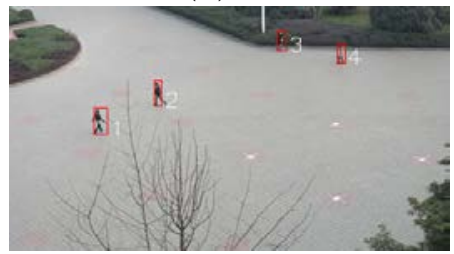

(b) frame 162

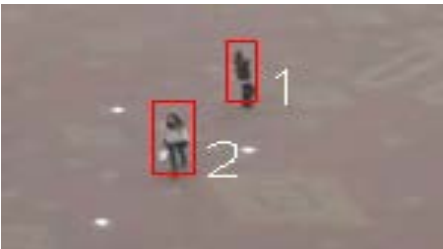

(c) frame 201

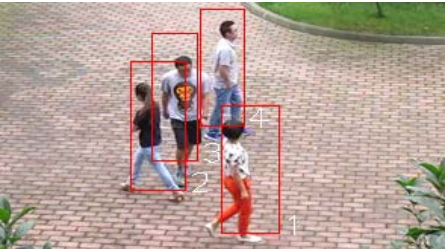

(c) frame 193

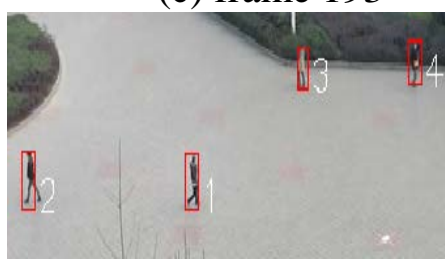

(c) frame 225

Analysis of Experimental Results

In order to verifying the effectiveness of the proposed method in the paper, we respectively tracking 50 video clips in different scenarios, the results are shown in Table 1,In the process of tracking,the matching degree of target box and target is little lower than the target tracking accuracy due to completely block or different block pattern ,In scenario 1 targets are large have accuracy direction estimation, so the tracking accuracy is high,the light conditions in scenario 2 is little beeter than scenario 1,3, but the target contains fewer pixels, so the accuracy will be lower than the scenario 1 and higher than 3.By the experimental results it can be seen that the method proposed by the paper has good tracking performance overall, and accuracy tracking within occlusions.

\begin{tabular}{|c|c|c|c|}
\hline Expriment scene & Videonumbers & Tracking accurate & Matching accurate \\
\hline Scenario 1 & 20 & $95.8 \%$ & $94 \%$ \\
\hline Scenario 2 & 15 & $94.2 \%$ & $93.3 \%$ \\
\hline Scenario 3 & 15 & $93.5 \%$ & $92.4 \%$ \\
\hline
\end{tabular}

Table 1. The analysis of experimental results

\section{Acknowledgement}

“Chunhui Plan” project number:Z2014050 


\section{Conclusions}

this paper proposes that use the optical flow to track pedestrians in partial occlusion and interaction area,with the combinnation of foreground segmentation result obtained by background modeling for consecutive frames based on mixture Gaussian model,as a constraint only the pixels in the foreground are for processing,uses the main optical flow direction energy as the feature to establish and update target model,when they have similiar direction ,the target position is predicted by its optical flow speed and direction.experimental results show that the algorithm of this paper has good and accuracy tracking performance within occlusions.But if objects are the same direction moving and sheltered,using this method can't reach accuracy target tracking,so the next step of the paper is combine the color feature, edge feature to establish a robust target model,for better multi-target tracking performance.

\section{References}

[1] D.M.Li ,T.Li :Multiple objects tracking based on mixture features,Advanced Materials Research, v 945-949, p 1869-1874, 2014

[2] Phadke,G:Robust Multiple Target Tracking Under Occlusion Using Fragmented Mean Shift and Kalman Filter,In International Conference on Communications and Signal Processing(ICCSP),2011

[3] Q.Wan,Y.N.Wang,and H.S.Yu:Tracking multiple persons under occlusion using appearance model based on attributed relational graph, Applied Mechanics and Materials, 2012,pp.1324-1328

[4] A.Alper,R.Michalis,Soatto,and Stefano,Sparse occlusion detection with optical flow,International Journal of Computer Vision.97(3)(2012)322-338

[5] S.Fazli,H.Moradi.Pour,and H.Bouzari:Particle Filter based Object Tracking with Sift and Color Feature.InSecond International Conference on Machine Vision,2009,Page(s): 89 - 93

[6] I.Haritaoglu,D.Harwood,and L.S.Davis.Hydra:Multiple people detection and tracking using silhouettes.In Second IEEE Workshop on Visual Surveillance,p. 6, Washington,DC,USA,1999.IEEE Computer Society

[7] Horn, B.K.P., Schunck, B.G.: Determining optical flow. Artificial Intelligence 17(1-3), 185-203 (1981)

[8] T.Yang, S.Z.Li, Q.Pan,and J.Li,Real-time Multiple Objects Tracking with Occlusion Handling in Dynamic Scenes,IEEE Computer Society Conference on Computer Vision and Pattern Recognition, CVPR 2005,pp.970-975

[9] Q.Wang, J.W.Fang, and Y.Yuan ,Multi-cue based tracking,Neurocomputing131(2014)227-236

[10] S.Denman,C.Fookes, and S.Sridharan,Group Segmentation During Object Tracking using Optical Flow Discontinuities,Fourth Pacific-Rim Symposium on Image and Video Technology (PSIVT)Image and Video Technology (PSIVT),2010

[11] J.Xu,A.Beaugendre,and S, Goto,A KLT-Based Approach for Occlusion Handling in Human Tracking,Picture Coding Symposium (PCS), 2012,Page(s): 337 - 340

[12] E.Parrilla, D.Ginestar, J.L.Hueso, J.Riera ,and J.R.Torregrosa,Handling occlusion in optical flow algorithms for object tracking,Computers \& Mathematics with Applications.56(3)(2008) 733-742

[13] D.Q.Sun,S.Roth,J.P.Lewis, and M. J. Black,Learning Optical Flow,Computer Vision - ECCV 2008, pp. 83-97

[14] S.Baker,D.Scharstein,J.P.Lewis ,S.Roth ,M.J.Black ,and R.Szeliski ,A Database and Evaluation Methodology for Optical Flow,11th International Conference on IEEE ,2007, Page(s):1-8 
[15] Lucas , B.D., Kanade, T.: An iterative image registration technique with an application to stereo vision. In: IJCAI, April 1981, pp. 674-679 (1981)

[16] S.Baker,D.Scharstein, J. P. Lewis, S. Roth, M. J. Black, and R. Szeliski.A database and evaluation methodology for optical flow. In Proc. ICCV,2007 